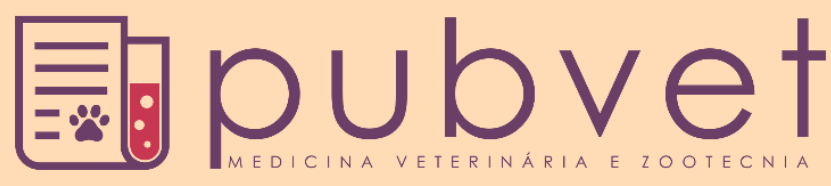

https://doi.org/10.31533/pubvet.v14n12a719.1-8

\title{
Toxocara spp., larva migrans visceral e saúde pública: Revisão
}

\author{
Dryellen Aparecida da Silva Felix ${ }^{1}$, Camila Xavier da Silva ${ }^{1}$, Jucélia da Silva Gomes ${ }^{1}$, Eduardo \\ Gomes Dias ${ }^{1}$, Jacelino da Silva Freitas ${ }^{1}$, Luis Eduardo dos Santos Fernandes ${ }^{1}$, Tamara de Moura \\ Mendes $^{2} \bullet$, Leonardo Alves de Farias ${ }^{30}$
}

${ }^{I}$ Discentes do curso de medicina veterinária da UNINASSAU, João Pessoa-PB, Brasil.

${ }^{2}$ Licenciada em Ciências Biológicas UFPB e discente do curso de Medicina veterinária da UNINASSAU, João Pessoa - PB, Brasil. ${ }^{3}$ Médico veterinário, mestre e docente do curso de Medicina veterinária da UNINASSAU, João Pessoa-PB, Brasil.

*Autor para correspondência, E-mail: dryellenaparecida17@gmail.com

\begin{abstract}
Resumo. As espécies Toxocara canis e Toxocara cati são responsáveis pela zoonose conhecida como toxocaríase, que afeta ocasionalmente o homem e são parasitas gastrintestinais de cães e gatos que apresentam distribuição global. No Brasil, o agente tem ampla distribuição, atingindo principalmente crianças, devido a hábitos geofágicos, contato íntimo com animais e acesso a lugares contaminados por fezes. Observa-se a necessidade de transferir informações à população, uma vez que essa importante infecção zoonótica está entre as mais comuns em todo o mundo e constitui um risco potencial à saúde. Em suma, esta revisão objetivou investigar os fatores predisponentes, os sinais clínicos, os meios de diagnóstico disponíveis e a prevalência da infecção em cães, gatos, canídeos e felídeos selvagens e o homem.
\end{abstract}

Palavras-chave: Toxocaríase, endoparasitas, helmintos, zoonose

\section{Toxocara spp., Larva migrans visceral and Public Health: Review}

Abstract. The species Toxocara canis and Toxocara cati are gastrointestinal parasites of dogs and cats that have a worldwide distribution and are responsible for the zoonosis known as toxocariasis, which occasionally affects humans. In Brazil, the agent has a wide distribution, mainly affecting children, due to geophagic habits, close contact with animals, and access to places contaminated by feces. There is a need to transfer information to the population since this important zoonotic infection is among the most common worldwide and constitutes a potential health risk. Thus, this review aimed to investigate the predisposing factors, clinical signs, available diagnostic methods, and the prevalence of infection in dogs, cats, canines and wild felids, and man, based on retrospective studies conducted in Brazil.

Keywords: Toxocariasis, endoparasites, helminths, zoonosis

\section{Toxocara spp., Larva migrans visceral y salud pública: Revisión}

Resumen. Las especies Toxocara canis y Toxocara cati son parásitos gastrointestinales de perros y gatos que tienen una distribución mundial y son responsables de la zoonosis conocida como toxocariasis, que afecta accidentalmente a los humanos. En Brasil, el agente tiene una amplia distribución, llegando principalmente a niños, debido a hábitos geofágicos, contacto cercano con animales y acceso a lugares contaminados por heces. Es necesario transferir información a la población, ya que esta importante infección zoonótica se encuentra entre las más comunes en todo el mundo y constituye un riesgo potencial para la salud. En resumen, esta revisión tuvo como objetivo investigar los factores predisponentes, los signos clínicos, los medios de diagnóstico disponibles y la prevalencia 
de infección en perros, gatos, caninos y felinos salvajes y hombres, según estudios retrospectivos realizados en Brasil.

Palabras clave: Toxocariasis, endoparásitos, helmintos, zoonosis

\section{Introdução}

A Toxocara canis é um nematódeo que pertence à família ascarídea, um grande verme de coloração creme, sendo a fêmea maior que o macho (Critchley et al., 1982; Mehlhorn, 2008; Overgaauw \& Boersema, 1998). Seu principal hospedeiro são os cães e demonstram um problema de saúde pública. A Toxocaríase é também conhecida por síndrome de larva migrans visceral, na grande maioria dos casos, comporta-se como uma doença benigna de curso limitado, embora existam casos graves e fatais (Chieffi et al., 1990; Peruca et al., 2012).

A infecção canina é mais frequente em cadelas prenhes e lactantes, bem como nos filhotes de 3 a 6 meses, já que a contaminação ocorre por via transplacentária e transmamária, O ciclo da larva migrans nos filhotes infectados acontece de três a quatro semanas após o nascimento, período em que ocorre a eliminação dos ovos de Toxocara canis para o meio ambiente a partir das fezes (Guimarães et al., 2005). O homem é um hospedeiro acidental pelo contato com os ovos em alimentos ou mesmo no manejo dos animais e seus utensílios (Pedrassani et al., 2008).

A prevalência desses parasitos e o contato da população com os mesmos são constantes, daí se tratar de uma zoonose, pois o aumento na taxa das populações canina e felina que frequentam o mesmo ambiente dos humanos é crescente. O alteroso contingente populacional de cães e gatos, a escassez de prevenção e controle de doenças e as condições desfavoráveis de vida dos animais aumenta o risco de transmissão. A prevalência desses parasitos em locais públicos ocasiona o aumento de contaminação e implicam na saúde humana, salientando assim a importância de meios profiláticos para recuperar um melhor parâmetro sanitário (Carvalho \& Rocha, 2011).

\section{Características morfológicas}

A Toxocara canis é um nematódeo que pertence à família ascarídea, um grande verme de coloração creme, sendo a fêmea maior que o macho (Mehlhorn, 2008). Seu principal hospedeiro são os cães. Os vermes machos medem em torno de 4 a $10 \mathrm{~cm}$, já as fêmeas medem de 9 a $18 \mathrm{~cm}$ de comprimento. Os estágios de desenvolvimento do parasita compreendem: ovos anembrionado, ovos embrionados, larvas no segundo, terceiro e quarto estágio (L2, L3 e L4), e verme adulto (Conceição et al., 2011).

O verme adulto reside no intestino delgado do cão. Sua Zoonose é a Larva Migrans Visceral. Seu corpo compõe-se na sua extremidade anterior de três lábios na boca, asas cervicais e tubo digestivo completo, na extremidade posterior apêndice terminal no macho e ânus na fêmea. $\mathrm{O}$ verme adulto da fêmea pode viver cerca de quatro a cinco meses no intestino do cão, nesse período são eliminados milhões de ovos (anembrionados) no solo, essa produção chega à média de 25.000 a 200.000 mil ovos por dia, que são secretados pelas fezes e passam em torno de duas semanas para tornarem-se embrionados. A partir do momento que migram no interior do hospedeiro sofrem trocas de cutícula externa do seu organismo que passam pelos estágios L3 é L4 até chegar então ao verme adulto (Conceição et al., 2011).

\section{Ciclo biológico}

Os cães filhotes se infectam pela ingestão de ovos contaminados contendo L2, em seguida, ocorre a penetração na mucosa intestinal, percorrendo adiante até a corrente sanguínea onde através da artéria aorta é distribuído aos mais diversos órgãos do hospedeiro formando grânulos (Fortes, 2004). Em cães adultos com o sistema imunológico desenvolvido, mesmo que as células imunitárias tenham mobilizado o parasito, pode ocorrer escape de algumas larvas que podem migrar para outros órgãos e tecidos, como músculos, rins e cérebro (Andrade et al., 2010).

Quando chegarem aos pulmões as larvas L2 saem dos vasos sanguíneos em direção ao tecido conjuntivo e entram nos alvéolos onde sofreram a muda para fase L3, migrando para os brônquios provocando tosse no animal, que as engolem novamente levando-as ao intestino. Desta forma, as larvas L3 chegam ao intestino delgado aonde vão mudar para o estado final L4, onde se desenvolvem em 
adultos e atingem 10 centímetros (Andrade et al., 2010). A migração que se segue está na dependência da idade e sexo do cão (Fortes, 2004). Após o amadurecimento genital, a fêmea de Toxocara canis é fecundada e inicia a postura de ovos. Em seguida, depositam milhares de ovos por dia nas fezes dos hospedeiros em condições ambientais favoráveis, desenvolvem-se em larva que sofrerão mudas tornando-se infectante para outros animais (Taylor et al., 2017).

Quando a infecção ocorre em cadelas gestantes às larvas são transmitidas através da placenta levando o parasito infectante (L2) ao fígado fetal, que perseguirá pelos pulmões de filhotes neonatos que dará continuidade pela migração traqueal. Logo, quase todos da cria nascem infectados, devido à migração transplacentária de larvas de Toxocara canis somáticos da cadela para o interior dos fetos (Conceição et al., 2011). Outra fonte de transmissão materna é após o parto pela amamentação, o parasito muda de L2 para L3, quando passa pelas glândulas mamárias, sendo ingerido na forma L3 pelos filhotes amamentados prosseguindo para faringe para dar continuidade ao ciclo (Conceição et al., 2011).

A Toxocara canis também é uma zoonose, quando os ovos infectantes são ingeridos pelo homem, ocorrendo migração pela via linfática ou circulação sanguínea. As L2 migarão ao fígado, sendo responsável pelo granuloma eosinófilo. Eventualmente podem atingir o globo ocular, ocasionando o deslocamento de retina. Estas larvas que não estão em seu hospedeiro adequado e não conseguem terminar seu ciclo evolutivo são denominadas de larva migrans visceral (LVM) (Bowman, 2004; Fortes, 2004; Taylor et al., 2017).

\section{Larva migrans visceral}

No gênero Toxocara há duas espécies que parasitam animais domésticos que podem infectar pessoas que convivem com cães e gatos, T. canis e T. cati, sendo a primeira mais comum (Quadros et al., 2014).

O Toxocara canis possui um padrão de migração larvária que lhe confere a capacidade de ser o agente etiológico mais envolvido na síndrome da larva migrans visceral. T. canis vive no intestino delgado de cães, gatos e canídeos silvestres. No período fértil a fêmea pode por cerca de 2 milhões de ovos por dia, diminuindo após o oitavo mês de vida. Os ovos desses parasitos são altamente resistentes no meio ambiente, toleram temperaturas entre $-10^{\circ} \mathrm{C}$ e $45^{\circ} \mathrm{C}$ e também à dessecação e umidade. Podem permanecer infectantes por até cinco anos, precisando de luz, umidade e oxigênio para completar seu desenvolvimento. Assim, os terrenos arenosos são mais favoráveis, pela sua elevada capacidade de oxigenação e drenagem. Os ovos que se encontram nas fezes, portanto, não estão embrionados e precisam completar o seu desenvolvimento no ambiente antes de embrionar e tornarem-se infectantes. Em condições de temperatura entre $15^{\circ} \mathrm{C}$ e $35^{\circ} \mathrm{C}$ e umidade, em torno de $85 \%$ dos ovos tornam-se infectantes entre duas e cinco semanas (Quadros et al., 2014).

O ciclo da infecção se inicia quando os ovos embrionados no solo são ingeridos e as larvas são liberadas no estômago e no intestino delgado, acometem a mucosa intestinal, o sistema linfático e, então, o fígado (Quadros et al., 2014). A larva atinge os pulmões e coração e migra para os tecidos somáticos, podendo, a partir desse estágio, ser transferido para o filhote no útero (infecção pré-natal) ou permanecer na forma "latente". Os cães podem se infectar de diversas formas, entre elas por migração transplacentária, pelo aleitamento ou pela ingestão de ovos infectantes (Quadros et al., 2014). Diferente do ancilostomídeo, este gênero não é hematófago e tem uma ação espoliativa no intestino.

O tratamento da toxocaríase baseia-se inicialmente na desinfecção dos cães acometidos e, posteriormente, na destruição e na eliminação do parasito Toxocara canis e também de seus ovos e larvas do organismo do animal. Os remédios utilizados no tratamento são anti-helmínticos e quase sempre são usados em filhotes. Seu uso em cães adultos possui efeito preventivo, uma vez que a toxocaríase nessa faixa etária acometem, em sua maioria, animais com o sistema imunológico enfraquecido. A prevenção consiste na higienização e desinfecção do local de permanência do cão, e na retirada das fezes diariamente do ambiente. Os ovos são muito resistentes a agentes químicos, necessitando muitas vezes de outros métodos, como vassoura de fogo (Quadros et al., 2014).

A infecção em cães é observada com maior incidência em cadelas prenhes e lactantes, assim como, em filhotes. Sendo as maiores infestações de parasitas observadas em filhotes de idade de três a seis meses. Nos filhotes de cães infectados as larvas completam o ciclo em três a quatro semanas após o 
nascimento do mesmo, sendo assim eles já são capazes de eliminar ovos de Toxocara canis para o meio ambiente junto com as fezes (Carvalho \& Rocha, 2011).

O homem participa do ciclo deste parasita de forma acidental. Ao ingerir alimentos que possuem os ovos contendo L3, ao chegarem ao intestino delgado do homem, liberam estas formas larvárias, que são capazes de atravessarem a mucosa intestinal e, por via linfática, atingirem a circulação e, por conseguinte, o fígado. As larvas saem do fígado caem na circulação sanguínea chegando aos pulmões. Atravessam os capilares pulmonares e caem na circulação pulmonar, em seguida no coração, disseminando-se para todo o organismo pela circulação sistêmica. Quando as larvas do T. canis excedem o diâmetro dos capilares sanguíneos, ocorre uma migração ativa e errática através da parede celular e dos tecidos do hospedeiro. A fase de migração ocasiona uma reação inflamatória aguda, com presença de eosinófilos, neutrófilos e, algumas vezes, monócitos. Também ocorre nesta fase há liberação de produtos metabolicamente ativos e antigênicos denominados antígenos de secreção-excreção. Foi demonstrado que esses antígenos se localizam na epicutícula das larvas e são receptores importantes para anticorpos (Lambertucci et al., 1996).

Em humanos a infecção por larvas de T. canis é em sua maioria assintomática. Quando sintomática atinge principalmente crianças. No entanto, os adultos também podem desenvolver a doença (Muradian et al., 2005).

\section{Ciclo de transmissão}

Os ascarídeos que causam toxocaríase no hospedeiro humano são o T. canis e o T. cati (Carvalho \& Rocha, 2011). Os vermes adultos vivem, em média, 4 meses e, em cerca de 6 meses, quase todos são eliminados espontaneamente pelo hospedeiro. A fêmea do T. canis produz até 200.000 ovos, que são resistentes a fatores hostis, podendo permanecer viáveis por tempo prolongado no solo. Os ovos nas fezes não são embrionados e, portanto, não são infectantes. Para que haja o embrionamento, são necessárias condições adequadas de temperatura $\left(15\right.$ a $\left.35^{\circ} \mathrm{C}\right)$ e umidade, sendo que, nessas condições, $85 \%$ dos ovos tornam-se infectantes no período de 2 a 5 semanas (Carvalho \& Rocha, 2011). O hospedeiro definitivo é o cão doméstico, no qual o parasita vive como adulto dentro do lúmen do intestino delgado. A história do T. canis depende da idade e do sexo do cão. Após o embrionamento sob condições adequadas do ambiente, cães podem infectar-se por vários meios (Carvalho \& Rocha, 2011). Ingestão de ovos infectantes: o cão ingere o ovo embrionado, que aparece nas fezes de 4 a 5 semanas após a infecção. Ingestão da larva e tecidos de hospedeiros paratênicos (minhoca, formiga e outros invertebrados que habitam o solo). Migração transplacentária: a prevalência do T. canis nos filhotes se aproxima dos $100 \%$. Passagem da larva pelo leite da cadela que amamenta seus filhotes: presença de larvas no colostro é máxima durante a $2^{\mathrm{a}}$ semana de lactação. Ingestão, pela cadela, de larvas do T. canis presentes nas fezes ou vômitos de filhotes, quando da higienização dos mesmos. A defecação pelos cães em praças públicas contribui para a contaminação ambiental com ovos de Toxocara, favorecendo a transmissão zoonótica. Estudos no Brasil verificaram ovos de parasita no solo a contaminação variou de 17,5, a 53,3\% (Carvalho \& Rocha, 2011).

A infecção na criança ocorre por ingestão dos ovos de T. canis por contaminação direta das mãos e, especialmente, dos dedos; contato direto com filhotes de cães, especialmente aqueles com idade entre 2 semanas e 6 meses; indiretamente, por contato com objetos contaminados com ovos infectados, dentro ou fora de casa; e por ingestão de terra, contendo larva ou ovos infectados (Carvalho \& Rocha, 2011).

A síndrome LMV é uma doença causada por ingestão de terra contendo larvas ou ovos infectados por T. canis. A geofagia não mostrou significância estatística para alguns autores. Alguns estudos relatam presença de pica (ingestão de substâncias não comestíveis) nas crianças com LMV; outros relatam a associação entre pica e toxocaríase. A pica é observada em muitas crianças com LMV, sendo prevalente entre as crianças entre 1 e 6 anos de idade (10 a 30\%), discretamente mais frequente em meninos do que em meninas. Prymak et al. (1988) mostraram a associação entre formas específicas de pica por fezes, terra ou cinzas e infecção pelo T. canis em 100 crianças entre 1 e 6 anos de idade. Um estudo de 2005 mostrou a associação entre sorologia positiva para T. canis e presença de cães filhotes e contato com terra entre crianças com idade média de 6,5 anos. Um trabalho não mostrou associação da onicofagia com sorologia positiva para T. canis, mas, em outro, a onicofagia foi fator de risco para toxocaríase (Carvalho \& Rocha, 2011). 
$\mathrm{O}$ fato de possuir cão na casa foi um fator de risco para sorologia positiva em vários estudos. Alguns autores não encontraram associação entre os donos de cães e a frequência de infecção por Toxocara, o que poderia ser explicado pelas medidas de higiene adequadas adotadas pelos adultos. Outros estudos mostraram associação de sorologia positiva para T. canis com filhotes menores de três meses (Carvalho \& Rocha, 2011).

Iddawela et al. (2003) mostraram que o padrão socioeconômico não foi significativo como fator de risco para toxocaríase. Outros autores mostraram associação significativa entre sorologia positiva e indicadores socioeconômicos, como baixo salário e nível de escolaridade. Vários autores encontraram alta prevalência em regiões com baixo poder aquisitivo, baixo índice de urbanização e parte da população sem acesso às condições sanitárias. As maiores taxas de soro prevalências estão associadas aos baixos níveis socioeconômico e/ou educacional. Em Brasília (DF), foi encontrada soro positividade para T. canis de $21,8 \%$ em amostras colhidas em laboratório de hospital público e de $3 \%$ em crianças de classe média, atendidas em laboratório privado. Alguns estudos mostraram associação entre morar em zona rural e toxocaríase (Carvalho \& Rocha, 2011).

A prevalência dos anticorpos de T. canis na população saudável mostra grande variabilidade territorial. Vários estudos mostraram soro prevalência variando entre 9,7 e $43 \%$. Outros trabalhos de descrição de casos isolados mostraram toxocaríase em crianças entre 16 meses e seis anos. Já outros estudos brasileiros, com maior tamanho amostral, apresentaram prevalência entre 1 e 14 anos. O mesmo foi encontrado em estudos realizados em outros países. Prymak et al. (1988) encontraram a média de idade de 5,6 anos. Nos países desenvolvidos, a prevalência foi maior em crianças de até 7 anos; porém, um estudo no Rio Grande do Sul mostrou toxocaríase em crianças entre 8 meses e 7 anos de idade. Em outros estudos brasileiros, a idade prevalente foi acima de 5 anos. Em estudos caso- controle, não foi encontrado diferença significativa em relação à idade (Carvalho \& Rocha, 2011).

\section{Saúde pública}

Com o aumento da densidade populacional nas grandes cidades e consequente aumento de cães e gatos, o ambiente urbano tem sido cada vez mais afetada pela contaminação ambiental e pela poluição fecal de jardins e parques públicos. Por todas estas razões, a Toxocaríase é atualmente a zoonose parasitária mais comum nos Países desenvolvidos (Otero et al., 2014).

Também os carnívoros silvestres têm revelados elevadas prevalência desta infecção, possivelmente potenciadas pela pressão humana sobre os seus habitats. O cão vem adquirindo cada vez mais, grande importância na sociedade moderna convivendo intimamente com o ser humano, entretanto, pode ser responsável pela transmissão de várias zoonoses. Cães levados pelos proprietários há locais de recreação pública, como praças, eliminam fezes e contaminam o solo dessas localidades com várias formas parasitárias (Santarém et al., 2004).

A contaminação de praças pública por fezes de cães constitui um problema de Saúde Pública, devido à possibilidade de transmissão de zoonoses, especialmente a Larva migrans visceral (LMV) e a Larva migrans cutânea (LMC) (Carvalho \& Rocha, 2011). A maioria dos estudos sobre a contaminação de praças públicas por ovos de helmintos de cães no Brasil enfocou a prevalência de Toxocara, devido à grande relevância da LMV. No entanto, em um surto de LMC em crianças de uma creche privada em Belo Horizonte, MG, a fonte de infecção foi uma caixa de areia contaminada por larvas de Ancylostoma. Desta forma, a transmissão desta zoonose também deve ser considerada em praças públicas contaminadas por fezes de cães, como foi observado em Campo Grande (Araújo et al., 1999).

Segundo Vinha (1965) o solo, com relação aos helmintos parasitas se comporta como um hospedeiro intermediário. Recebe fezes ou água contaminada por parasitas em estágios não infectantes, oferecelhes condições para o desenvolvimento, os protege durante certo tempo na fase infectante para, posteriormente, transmiti-lo ao homem. Ao defecar no solo o cão infectado libera milhões de ovos, que em condições ambientais favoráveis, como umidade e temperatura, podem eclodir e desenvolver-se em larvas de terceiro estágio (L3), sendo essa a forma infectante. Essa larva é o principal agente etiológico da migrans cutânea (LMC) no homem, onde a larva migra entre a derme e epiderme, podendo causar lesões eritêmato-populosas ou urticariformes, muito pruriginosas, hemorragias petequeais e edema 
inflamatório. Nesse caso não ocorre o desenvolvimento do parasito adulto, pelo fato do homem não ser o hospedeiro adulto normal (Bowman, 2014; Neves, 2005).

Em situações ambientais adversas, ocorre o desenvolvimento de clamidósporos, estruturas semelhantes aos esporos, mas com uma parede espessa e resistente e com uma reserva nutritiva. Já em condições favoráveis, o clamidósporo dá origem a uma nova hifa, permitindo, desta forma, a sobrevivência do fungo (Larone, 1995). Estes fungos atuam nos ovos e larvas dos parasitas presentes no solo, destruindo-os, e quebrando assim o ciclo de vida do parasita. Como os esporos fúngicos não produzem efeitos nocivos no ambiente ou animal, e como resistem ao processo digestivo, é possível administrá-los oralmente, sendo eliminados juntamente com as fezes para o solo onde irão atuar (Braga \& Araújo, 2014; Yacob et al., 2009). Um dos fatores que aumentam o risco da contaminação desta enfermidade é o tamanho da população canina de certa região (Fortes, 2004; Urquhart, 1996).

Segundo Neves (2005), a LMV é uma infecção humana muito frequente em crianças, que se contaminam pela ingestão de ovos larvados, fômites e mãos contaminadas. Para descontaminação do solo e controle ambiental podem ser utilizadas diferentes metodologias, tais como: cobertura do solo com lâminas de vinil para destruição dos ovos de Toxocara spp. (nas regiões com temperaturas superiores a $30^{\circ}$ e durante um mínimo de 3 horas) (Uga \& Kataoka, 1995); aplicação de fontes de calor, utilizando uma temperatura superior a $60^{\circ} \mathrm{C}$ durante 5 minutos (Bowman, 2004); exposição à luz ultravioleta; e solução aquosa iodada (Overgaauw \& van Knapen, 2013). Para melhorar esse parâmetro sanitário, é necessário realizar programas educacionais, para a conscientização sobre a importância da erradicação desses parasitos por meio de tratamento dos animais, medidas de restrição física dos parques e melhoria das condições de saneamento básico. Paralelo a isso, é importante a participação dos gestores na definição de políticas públicas, objetivando o controle dessas e de outras parasitoses (․ㅡㄹ et al., 2019).

\section{Considerações finais}

A prevalência desses parasitos e o contato da população humana com os mesmos são constantes, devido ao aumento na taxa das populações canina e felina que frequentam o mesmo ambiente dos humanos. As atitudes para mudanças deste cenário não devem ser isoladas ou apenas dependentes do Poder Público. É de suma importância um esforço conjunto da sociedade, Médicos Veterinários e demais profissionais da Saúde Única, que, por meio da educação para guarda responsável e realização de programas educacionais de conscientização sobre a importância da erradicação desses parasitos através do tratamento dos animais acometidos, bem como medidas de restrição física dos parques e melhoria das condições de saneamento básico, seja possível, inicialmente, a redução e, finalmente o controle deste problema que afeta a todos, promovendo assim a garantia à saúde, à segurança e o conforto dos animais. Espera-se que, pelo presente estudo, este possa contribuir para uma melhor aprendizagem e interpretação sobre as síndromes LMC e LMV alem de fornecer embasamento teórico e científico para futuros estudos sobre o tema abordado.

\section{Referências bibliográficas}

Andrade, E. C., Leite, I. C. G., Oliveira Rodrigues, V., \& Cesca, M. G. (2010). Parasitoses intestinais: uma revisão sobre os seus aspectos sociais, epidemiológicos, clínicos e terapêuticos. Revista de APS, 13(2), 231-240.

Araújo, F. R., Crocci, A. J., Rodrigues, R. G. C., Silva Avalhaes, J., Miyoshi, M. I., Salgado, F. P., Silva, M. A., \& Pereira, M. L. (1999). Contaminação de praças públicas de Campo Grande, Mato Grosso do Sul, Brasil, por ovos de Toxocara e Ancylostomaem fezes de cães. Revista Da Sociedade Brasileira de Medicina Tropical, 32(5), 581-583. DOI: https://doi.org/10.1590/s0037$\underline{86821999000500017}$

Bowman, D. (2004). Parasitologia veterinária de Georgis. Elsevier Brasil.

Bowman, D. D. (2014). Georgis' Parasitology for Veterinarians-E-Book. Saunders Company.

Braga, F. R., \& Araújo, J. V. (2014). Nematophagous fungi for biological control of gastrointestinal nematodes in domestic animals. Applied Microbiology and Biotechnology, 98(1), 71-82. DOI: https://doi.org/10.1007/s00253-013-5366-z

Carvalho, E. A. A., \& Rocha, R. L. (2011). Toxocaríase: larva migrans visceral em crianças e 
adolescentes. Jornal de Pediatria, 87(2), 100-110. DOI: https://doi.org/10.2223/JPED.2074

Chieffi, P. P., Ueda, M., Camargo, E., Souza, A., Guedes, M., Gerbi, L., Spir, M., \& Moreira, A. (1990). Visceral larva migrans: A seroepidemiological sunvsv in five municipalities of São Paulo state, Brazil. Revista Do Instituto de Medicina Tropical de São Paulo, 32(3), 204-210. DOI: https://doi.org/10.1590/s0036-46651990000300010

Conceição, L. G., Acha, L. M. R., Borges, A. S., Assis, F. G., Loures, F. H., \& Silva, F. F. (2011). Epidemiology, clinical signs, histopathology and molecular characterization of canine leproid granuloma: a retrospective study of cases from Brazil. Veterinary Dermatology, 22(3), 249-256. DOI: https://doi.org/10.1111/j.1365-3164.2010.00934.x

Critchley, E. M. R., Vakil, S. D., Hutchinson, D. N., \& Taylor, P. (1982). Toxoplasma, toxocara, and epilepsy. Epilepsia, 23(3), 315-321. DOI: https://doi.org/10.1111/j.1528-1157.1982.tb06197.x

Fortes, E. (2004). Parasitologia veterinária (4th ed.). Editora ícone.

Guimarães, A. M. ., Alves, E. G. L., \& Rezende, G. F. (2005). Ovos de Toxocara sp. e larvas de Ancylostoma sp. em praça pública de Lavras, MG. Revista de Saúde Pública, 39(2), 293-295. DOI: https://doi.org/10.1590/s0034-89102005000200022

Iddawela, D. R., Kumarasiri, P. V, \& Wijesundera, M. S. (2003). A seroepidemiological study of toxocariasis and risk factors for infection in children in Sri Lanka. Southeast Asian Journal of Tropical Medicine and Public Health, 34(1), 7-15.

Lambertucci, J. R., Gerspacher-Lara, R., Pinto-Silva, R. A., Barbosa, M. M., Teixeira, R., Barbosa, H. F., Serufo, J. C., Rezende, D. F., Drummond, S. C., \& Rayes, A. A. M. (1996). O projeto Queixadinha: a morbidade e o controle da esquistossomose em área endêmica no nordeste de Minas Gerais, Brasil. Revista Da Sociedade Brasileira de Medicina Tropical, 29(2), 127-135. DOI: https://doi.org/10.1590/s0037-86821996000200005

Larone, D. H. (1995). Medically important fungi- A guide to identification. ASM Press.

Mehlhorn, H. (2008). Encyclopedia of parasitology. Springer.

Muradian, V., Gennari, S. M., Glickman, L. T., \& Pinheiro, S. R. (2005). Epidemiological aspects of visceral larva migrans in children living at Sao Remo Community, São Paulo (SP), Brazil. Veterinary Parasitology, 134(1-2), 93-97. DOI: https://doi.org/10.1016/j.vetpar.2005.05.060

Neves, D. P. (2005). Parasitologia humana. Editora Atheneu.

Otero, D., Nijsse, R., Gomes, L., Alho, A., Overgaauw, P., Hoek, D., \& Carvalho, L. M. (2014). Prevalência de ovos de Toxocara spp. no solo de parques públicos da área da grande Lisboa, Portugal—resultados preliminares. Acta Parasitológica Portuguesa, 20(1/2), 47-50.

Overgaauw, P. A. M., \& Boersema, J. H. (1998). A survey of Toxocara infections in cat breeding colonies in the Netherlands. Veterinary Quarterly, 20(1), 9-11.

Overgaauw, P. A. M., \& van Knapen, F. (2013). Veterinary and public health aspects of Toxocara spp. Veterinary Parasitology, 193(4), 398-403. DOI: https://doi.org/10.1016/j.vetpar.2012.12.035

Pedrassani, D., Viera, A. M., \& Thiem, E. M. B. (2008). Contaminação por Toxocara spp. e Ancylostoma spp. em áreas de lazer do município de Canoinhas, SC. Archives of Veterinary Science, 13(2), 110-117. DOI: https://doi.org/10.5380/avs.v13i2.12895

Peruca, L. C. B., Langoni, H., \& Lucheis, S. B. (2012). Larva migrans visceral e cutânea como zoonoses: revisão de literatura. Veterinária e Zootecnia, 16(4), 601-616.

Prymak, C., McKee, L. J., Goldschmidt, M. H., \& Glickman, L. T. (1988). Epidemiologic, clinical, pathologic, and prognostic characteristics of splenic hemangiosarcoma and splenic hematoma in dogs: 217 cases (1985). Journal of the American Veterinary Medical Association, 193(6), 706-712.

Quadros, R. M., Liz, F. R., \& Marques, S. M. T. (2014). Ocorrência de ovos de Toxocara spp. em solos de praças públicas de Lages, Santa Catarina. ARS Veterinaria, 30(2), 109-114. DOI: https://doi.org/10.15361/2175-0106.2014v30n2p109-114

Rocha, M. J. da, Weber, D. M., \& Costa, J. P. da. (2019). Prevalência de larvas migrans em solos de parques públicos da cidade de Redenção, estado do Pará, Brasil. Revista Pan-Amazônica de Saúde, 10, 1-8. DOI: https://doi.org/10.5123/s2176-6223201901607 
Santarém, V. A., Giuffrida, R., \& Zanin, G. A. (2004). Larva migrans cutânea: ocorrência de casos humanos e identificação de larvas de Ancylostoma spp em parque público do município de Taciba, São Paulo. Revista Brasileira de Medicina Tropical, 37(2), 179-181. DOI: https://doi.org/10.1590/s0037-86822004000200014

Taylor, M. A., Coop, R. L., \& Wall, R. L. (2017). Parasitologia Veterinária. Guanabara Koogan.

Uga, S., \& Kataoka, N. (1995). Measures to control Toxocara egg contamination in sandpits of public parks. The American Journal of Tropical Medicine and Hygiene, 52(1), 21-24. DOI: https://doi.org/10.4269/ajtmh.1995.52.21

Urquhart, G. M. (1996). Parasitologia veterinária (2nd ed.). Guanabara Koogan.

Vinha, C. (1965). Fundamentos e importância das campanhas contra os geo-helmintos no Brasil. Revista Brasileira de Malariologia e Doenças Tropicai, 17, 379-406.

Yacob, H. T., Mistre, C., Adem, A. H., \& Basu, A. K. (2009). Parasitological and clinical responses of lambs experimentally infected with Haemonchus contortus (L3) with and without ivermectin treatment. Veterinary Parasitology, 166(1-2), 119-123. DOI: http://dx.doi.org/10.1016/j.vetpar.2009.07.038

Histórico do artigo:

Recebido: 14 de maio, 2020.

Aprovado: 23 de junho, 2020.

Disponível online: 9 de novembro, 2020
Licenciamento: Este artigo é publicado na modalidade Acesso Aberto sob a licença Creative Commons Atribuição 4.0 (CC-BY 4.0), a qual permite uso irrestrito, distribuição, reprodução em qualquer meio, desde que o autor e a fonte sejam devidamente creditados. 